\title{
A Thermal Balancing Analysis of Composites Reinforced by Different Shape Buckypaper
}

\author{
Aying Zhang ${ }^{1, a,{ }^{*}}$, Zhenghong $\mathrm{Li}^{2, b}$ \\ ${ }^{1}$ Harbin University, 150086 Harbin, China \\ ${ }^{2}$ Harbin Institute of Technology, 150001 Harbin, China \\ a,*zaying@sina.com, b273662999@qq.com
}

Keywords: Thermal Balancing Analysis, Simulation, Buckypaper, Composites.

\begin{abstract}
The software FLUENT was used to analysis the thermal balance of composites reinforced by different shape buckypaper during the heating process. The research shows that the electrothermal energy is mainly used to increase the temperature of the insulator and the heating plate itself at the initial stage of heating. Because the mass and heat capacity of the insulators are both greater than that of the heating plates, the insulators absorb more heat than the heating sheets. The heating rate of insulators and heating sheets slows down as time increases, which result in the decrease of heat absorption. The increase of external surface temperature makes convection heat dissipation increase, so the proportion of convection heat dissipation increases, and the endothermic ratio of material decreases.
\end{abstract}

\section{Introduction}

Compared with graphite films, buckypaper may exhibit a better thermal conducting performance not only in the plane but also more importantly along the direction perpendicular to the plane of buckypaper, i.e., along the thickness direction. Wang et al [1] reported a mechanical process by which high-quality buckypapers (BPs) could be prepared by rolling a cylindrical pin over arrays of multiwalled carbon nanotubes (MWCNTs). The aligned orientation of the MWCNTs in their samples improved room temperature values of electrical conductivity $(\sigma)$ by a factor of 1.3 and of $\kappa$ by a factor of 2. Zhang et al [2] reported electrical and thermal properties of a CNT bulk material made of randomly oriented MWCNTs by spark plasma sintering (SPS) at $1600{ }^{\circ} \mathrm{C}$. Their results showed rich transport phenomena which the authors interpreted in terms of variable range hopping and fluctuation assisted tunneling [2].

Thin sheets comprised of randomly oriented MWCNTs, commonly referred as buckypaper (MWCNT-BP), are expected to exhibit excellent electrical and thermal properties in view of the exceptionally high electrical $\left(\sim 10^{-6} \mathrm{~m} \Omega \bullet \mathrm{cm}\right)$ and thermal $(\sim 3000 \mathrm{~W} /(\mathrm{m} \bullet \mathrm{K}))$ conducting properties of individual MWCNTs [3,4]. Carbon nanotube (CNT) buckypaper, which has large specific surface area and tunable network structures, shows great potential in the application of heat dissipation for high power electronic devices. Chen et al [5] investigated the effects of CNT diameter and length on the network formation of buckypapers by vacuum filtration and their thermal transport properties were thoroughly. The CNT architecture in buckypaper is found to be a key issue to affect the thermal conductivity of buckypapers.

In this study, the heating model of the polymer composite reinforced by flat, sinusoidal and pulse bending buckypaper is established to predict the thermal property of buckypaper reinforced composite. The finite element software FLUENT is used to analyze how the bending shapes affect the thermal balance. 


\section{Numerical model}

The software FLUENT was used to analysis the thermal balance of composites reinforced by different shape buckypaper during the heating process. Figure 1 shows the heating model of the polymer composite reinforced by the buckypaper.

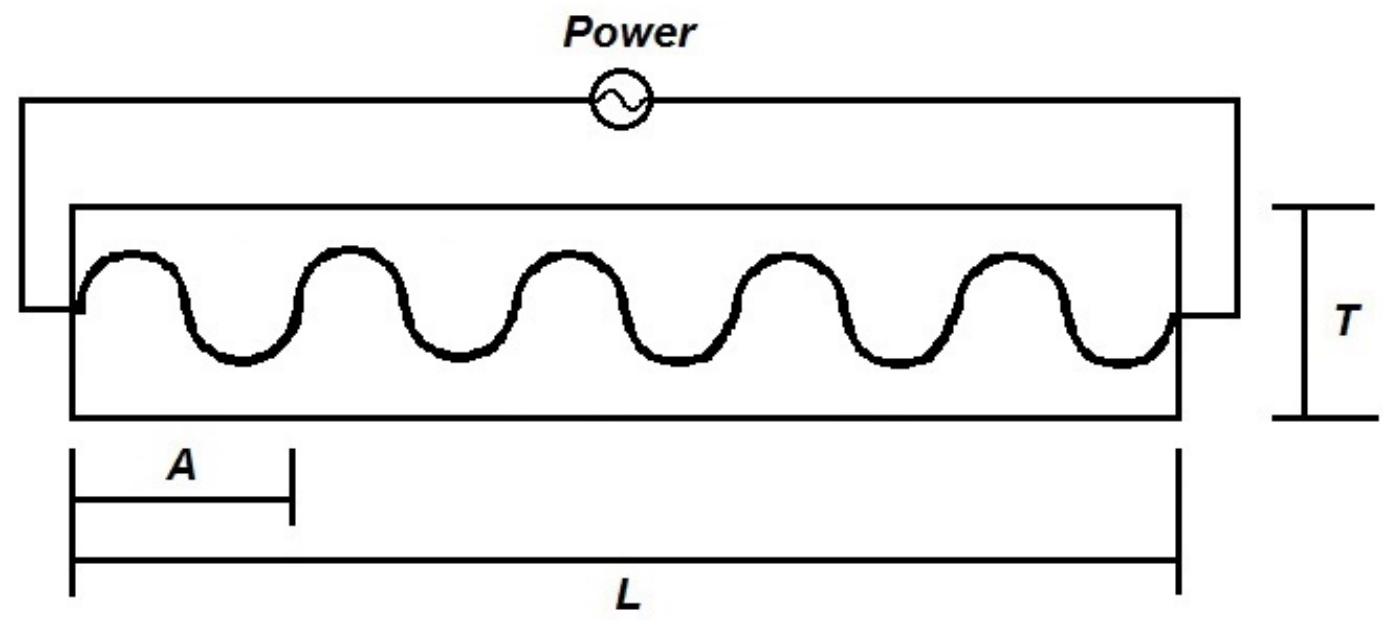

Fig. 1. Sketch diagram of heating experimental device

As shown in Figure 1, the length $(L)$ and the thickness $(T)$ of the heating model are $600 \mathrm{~mm}$ and $100 \mathrm{~mm}$ respectively, and the width $(w)$ is $50 \mathrm{~mm}$. The thicknesses of the buckypaper are $10 \mathrm{~mm}$. The bending height $(h)$ and bending period $(A)$ of the sinusoidal and pulse bending buckypaper are $60 \mathrm{~mm}$ and $120 \mathrm{~mm}$. The heating powers are $25 \mathrm{~W}$.

\section{Calculation Condition}

It is known by the conservation of energy, the energy conservation can be divided into three parts from the beginning of heating to the steady state. The increasing part of the internal energy of the insulation body and the heating sheets, and the external heat dissipation part of the system.

As shown in formula (1).

$$
Q_{\text {total }}=\Delta U_{\text {heater }}+\Delta U_{\text {insulator }}+Q_{\text {loss }}
$$

Among them, the total heating energy $Q_{\text {total }}$ consumption can be obtained by constant heating power and the time needed to achieve steady state. The increase of the internal energy of the two materials can be expressed as formula (2).

$$
\Delta U=\int \rho \cdot c_{p} \cdot(\delta T)_{v} \mathrm{~d} v=\rho c_{p} \int\left(T_{v}-T_{0}\right) \mathrm{d} v=\rho c_{p}\left(T_{\text {ave }}-T_{0}\right) V
$$

As shown in formula (3), $V$ is the total volume of each material, $T_{0}$ is the initial temperature, and $T_{\text {ave }}$ is the volume average temperature.

$$
T_{\text {ave }}=\int T_{v} \mathrm{~d} v / V
$$

The volume average temperature of insulators $\Delta U_{\text {insulator }}$ and heating sheets $\Delta U_{\text {heater }}$ can be obtained when the steady state is derived respectively. The system dissipate heat through convection on the outside surface and ambient air. The total heat dissipation is difficult to obtain directly as the surface temperature changes continuously during the heating process. The total amount of heat lost $Q_{\text {loss }}$ during the heating process can be obtained by replacing the internal energy added material and the total consumption heat into the conservation of energy. The ratio of total heat consumption to each heat input can be calculated after obtaining the heat flows back to each part from the beginning of heating to a certain moment. 


\section{Results and discussion}

Figures 2 shows that the ratio of the total heat consumption to the total heat consumption vary with the heating time under the shape of the three heating plates. The thermal conductivity of buckypaper was set to be $1.0 \mathrm{~W} /(\mathrm{m} \cdot \mathrm{K})$. The thermal conductivity of the polymer matrix was set to be 0.1 $\mathrm{W} /(\mathrm{m} \cdot \mathrm{K})$.

As shown in Figures 2, the electrothermal energy is mainly used to increase the temperature of the insulator and the heating plate itself at the initial stage of heating. Because the mass and heat capacity of the insulators are both greater than that of the heating plates, the insulators absorb more heat than the heating sheets. The heating rate of insulators and heating sheets slows down as time increases, which result in the decrease of heat absorption. The increase of external surface temperature makes convection heat dissipation increase, so the proportion of convection heat dissipation increases, and the endothermic ratio of material decreases.

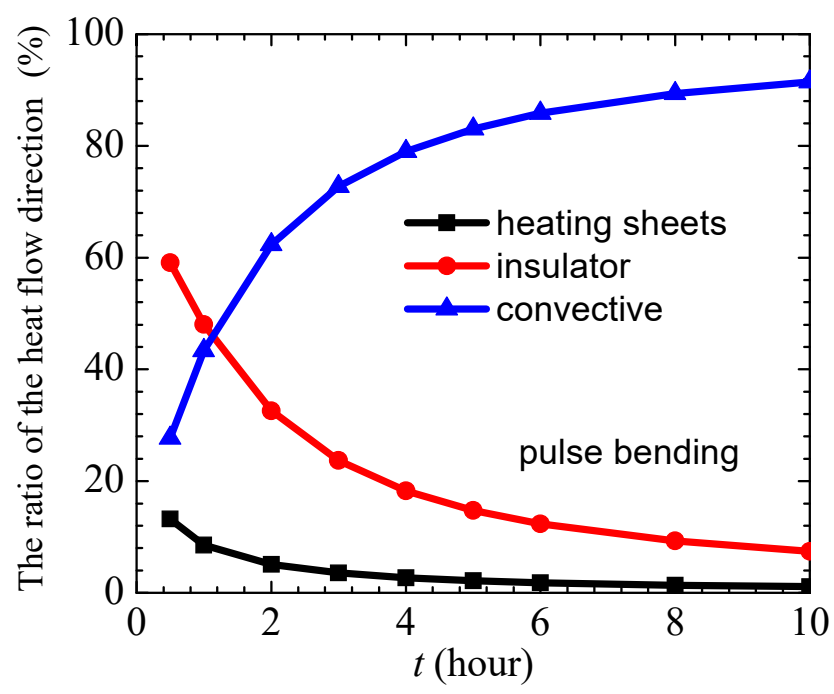

(a) pulse bending

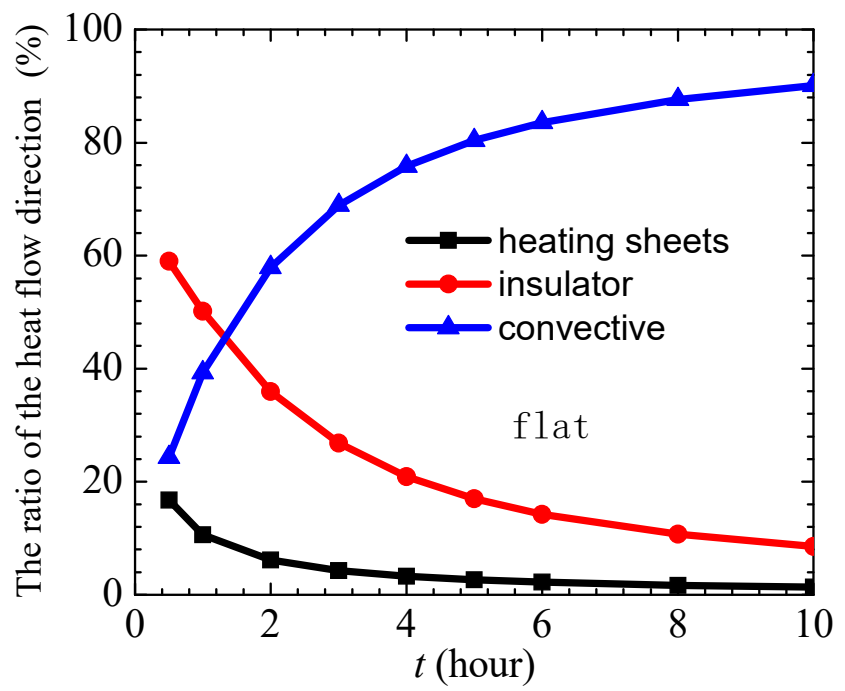

(b) flat 


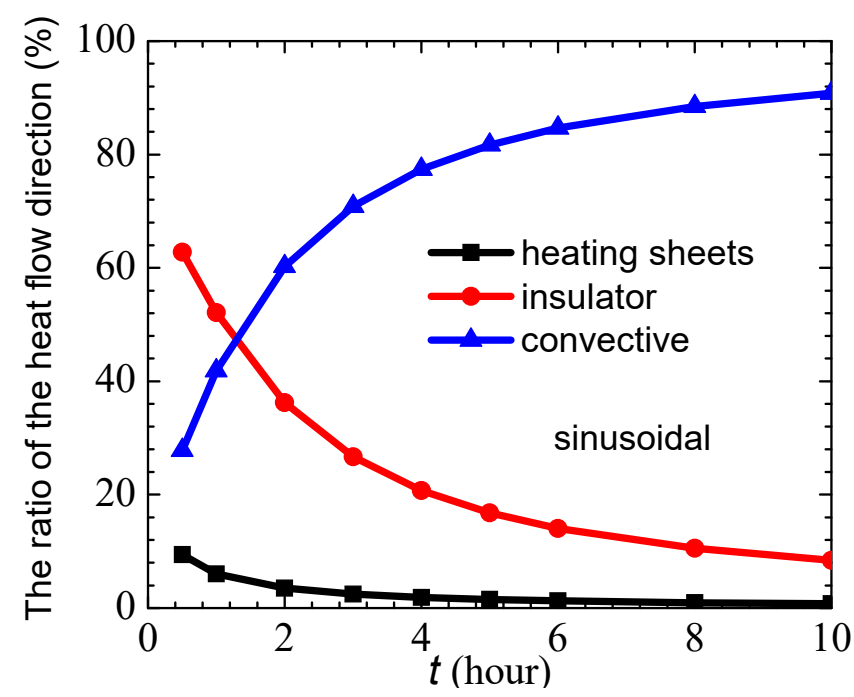

(c) sinusoidal

Fig. 2. The ratio of the heat flow direction vary with the heating time

\section{Summary}

The software FLUENT was used to analysis the thermal balance of composites reinforced by different shape buckypaper during the heating process. The research shows that the electrothermal energy is mainly used to increase the temperature of the insulator and the heating plate itself at the initial stage of heating. Because the mass and heat capacity of the insulators are both greater than that of the heating plates, the insulators absorb more heat than the heating sheets. The heating rate of insulators and heating sheets slows down as time increases, which result in the decrease of heat absorption. The increase of external surface temperature makes convection heat dissipation increase, so the proportion of convection heat dissipation increases, and the endothermic ratio of material decreases.

\section{Acknowledgement}

This research was financially supported by Heilongjiang Natural Science Foundation (Grant No. E201454).

\section{References}

[1] D. Wang, P. Song, C. Liu and et al, Highly oriented carbon nanotube papers made of aligned carbon nanotubes, Nanotechnology, vol. 7, pp. 075609, 2008.

[2] D. Wang, P. Song, C. Liu, W. Wu and et al, Highly oriented carbon nanotube papers made of aligned carbon nanotubes, Nanotechnology, vol. 7, pp. 369-381, 2008.

[3] P. Kim, L. Shi, A. Majumdar and et al, Thermal transport measurements of individual multiwalled nanotubes, Physical Review Letters, vol. 87, pp. 265-266, 2001.

[4] H. J. Li, W. G. Lu, J. J. Li and et al, Multichannel ballistic transport in multiwall carbon nanotubes, Physical Review Letters, vol. 8, pp. 086601, 2005.

[5] Hongyuan Chen, Minghai Chen and et al, Architecting Three-Dimensional Networks in Carbon Nanotube Buckypapers for Thermal Interface Materials, The Journal of Physical Chemistry C, vol. 6, pp. 3903-3909, 2012. 